Military Technical College Kobry El-Kobba, Cairo, Egypt

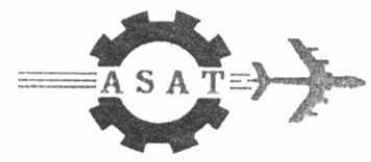

11-th International Conference on Aerospace Sciences \& Aviation Technology

\title{
PERFORMANCE OF TURBO CODES WITH SPACE-TIME TRANSMIT DIVERSITY
}

\author{
Salah, M. M., El-Agooz, S. S., Mahroos, A. I."
}

\begin{abstract}
In future wireless communication systems such as $3^{\text {rd }}$ generation mobile systems, increasing the downlink capacity from the base stations to remote units becomes more important due to high data rate applications such as wireless internet access. Turbo Codes and Space-Time Transmit Diversity (STTD) are two of the most recently techniques to improve system performance and capacity. In this paper, the performance of turbo codes with transmit diversity is studied. The effect of number of transmits antennas, puncturing, and frame length are investigated over Rayleigh fading channels. The simulation results show that a significant gain is achieved when using turbo codes with space-time transmit diversity technique.
\end{abstract}

KEYWORDS: Turbo Codes, Space-Time Codes, Diversity

\footnotetext{
* Egyptian Armed Forces
} 


\section{INTRODUCTION}

Turbo codes, also known as parallel concatenated convolutional codes (PCCC), are the most exciting and important development in coding theory in recent years. They were first introduced by Berrou et al. in 1993 [1]. Turbo codes combine recursive systematic convolutional (RSC) codes along with a pseudorandom internal interleaver and maximum a posteriori probability (MAP) iterative decoding algorithm to achieve performance very close to the Shannon limit [1]. Due to the delay introduced by the turbo internal interleaver, turbo codes are more suitable for data transmissions. Turbo codes have been adopted in the 3rd generation mobile systems, such as WCDMA, mainly for the high data rate applications [2]. A study of the performance and analysis of turbo codes over Rayleigh fading channels is presented in $[3,4]$.

The wireless mobile communication systems are sensitive to time-varying impairments, such as multipath fading and interference. Achieving high data rates over wireless channels is a challenging task. In general, time-varying fading due to multipath propagation can be effectively combated by employing various diversity techniques, including time, frequency, and space diversity.

Interleaving is a way to break the channel temporal correlation and provide time diversity. However, the interleaving depth is usually limited by the interleaving delay constraints and memory requirement.

The multiple antennas on transmitter or receiver provide the space diversity. It might be difficult, however, to have multiple antennas on the mobile handset due to the limitations on power, size and weight of mobile handsets. Hence, from the engineering point of view, it makes sense to place multiple antennas and additional complexity on base stations, which have fixed location and sufficient processing and energy resources compared to the mobile handsets.

Transmit diversity techniques provide a relatively low cost solution to increase downlink capacity in 3rd generation mobile systems [8]. There has been a lot of work dedicated to the study of transmit diversity techniques [5]. A transmit diversity scheme for two transmit antennas and a simple decoding algorithm was introduced by Alamouti in [5] and generalized to an arbitrary number of transmit antennas as Space-Time Block Coding by Tarokh et al. in [6]. Space-time block codes are designed to achieve the maximum possible diversity order of $n_{T} . n_{R}$ for $n_{T}$ transmit and $n_{R}$ receive antennas under the constraint of having a simple decoding algorithm [9]. Furthermore, for a certain diversity advantage they provide the minimum possible decoding delay. However, space-time block codes are not designed to achieve an additional coding gain. Therefore, space-time block codes should be concatenated with an outer code like Turbo Codes [10] which provides a significant coding gain. In this paper, the term space time transmit diversity (STTD) is used specially to denote the space time block coding scheme [5]. Turbo coding and downlink transmit diversity are two of the most recently techniques in improving the downlink capacity. 
From the study of space-time processing [8], the large capacity gain from space diversity is achieved especially when the transfer functions between different transmit and receiver antenna pairs is uncorrelated.

In this paper, it is assumed that the antennas at the base station are placed apart from each other with large enough distance and the receiver has a perfect acknowledge of channel state information (CSI).

The paper is organized as follows. In the next section, the theory of turbo decoding algorithms is summarized. In section III, the space-time transmit diversity technique is illustrated. In section IV, The system model of a turbo codes with space time coding is described. In section $\mathrm{V}$, the simulation results on the performance of turbo codes with downlink transmit diversity over Rayleigh fading channels are presented. Finally, the conclusion is provided in section VI.

\section{TURBO DECODING ALGORITHM}

The general structure of an iterative turbo decoder is shown in Figure 1. Two component decoders are linked by interleavers in a structure similar to that of the encoder. As seen in the figure, each decoder takes three inputs - the systematically encoded channel output bits, the parity bits transmitted from the associated component encoder, and the information from the other component decoder ( extrinsic ) about the likely values of the bits concerned. The information from the other decoder is referred to as a-priori information. The component decoders have to exploit both the inputs from the channel and this a-priori information. They must also provide what are known as soft outputs for the decoded bits. This means that as well as providing the decoded output bit sequence, the component decoders must also give the associated probabilities for each bit that it has been correctly decoded. The soft outputs are typically represented in terms of the so-called Log Likelihood Ratios (LLRs), the magnitude of which gives the sign of the bit, and the amplitude provides the probability of correct decision. A suitable decoder is the Maximum A-Posteriori (MAP) [11] algorithm.

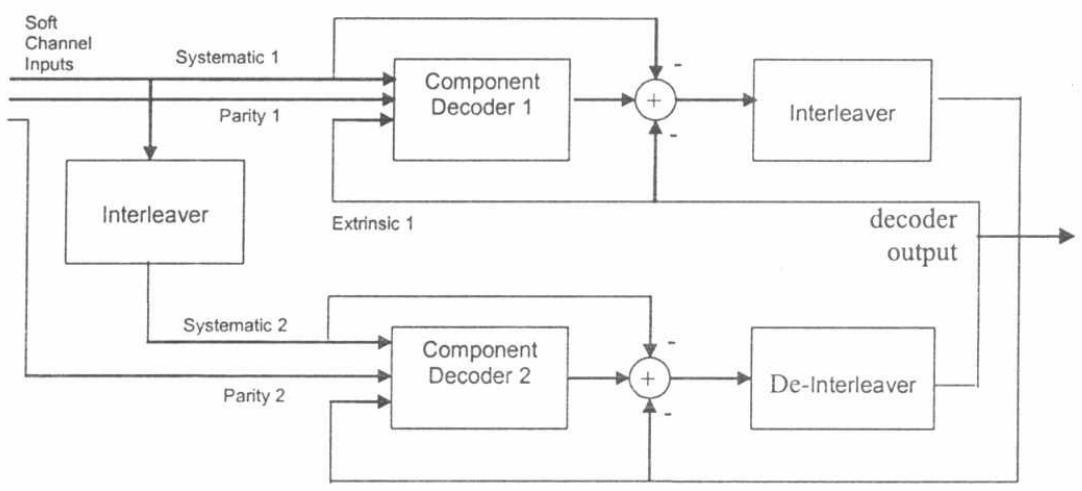

Fig. 1. Iterative turbo decoding scheme using the SISO algorithm. 


\section{SPACE-TIME TRANSMIT DIVERSITY}

With Alamouti's transmit diversity technique [5], in any given transmission period two signals are transmitted simultaneously from two transmit antennas. The encoder takes two symbols $x_{1} x_{2}$ at a time. Signal $x_{1}$ is transmitted from the first transmit antenna while signal $x_{2}$ from the second one. In the next transmission instance, signal $-x_{2}{ }^{*}$ is transmitted from the first transmit antenna and signal $x_{1}{ }^{*}$ from the second one, where $x_{i}^{*}$ is the complex conjugate of $x_{i}, \mathrm{i} \in\{1,2\}$. Assuming that one receive antenna is employed and that the fading coefficients are constant across two consecutive symbols, the channel fading gains $h_{1}(t)$ and $h_{2}(t)$, for the first and the second transmit antennas,

respectively, can be expressed as follows:

$$
\begin{aligned}
& h_{1}(t)=h_{1}(t+T)=h_{1}=\left|h_{1}\right| e^{j \theta_{1}} \\
& h_{2}(t)=h_{2}(t+T)=h_{2}=\left|h_{2}\right| e^{j \theta_{2}}
\end{aligned}
$$

where $\left|h_{i}\right|, \mathrm{i} \in\{1,2\}$ is the channel gain from transmit antenna $\mathrm{i}$ to the receive antenna, $T$ is the symbol duration, and $\theta_{i}$, is the channel phase shift from transmit antenna $i$ to the receive antenna. At the receive antenna, the received signals $r_{1}$ and $r_{2}$ at time $\mathrm{t}$ and $\mathrm{t}+\mathrm{T}$, respectively, can be expressed as,

$$
\begin{aligned}
& r_{1}=h_{1} x_{1}+h_{2} x_{2}+n_{1} \\
& r_{2}=-h_{2} x_{2}^{*}+h_{2} x_{1}^{*}+n_{2}
\end{aligned}
$$

where $n_{i}, \mathrm{i} \in\{1,2\}$ is a random variable representing a sample of additive white Gaussian noise with one sided power spectral density of $N_{o}$ per dimension at time $t+(i-1) T$.

At the receiver, a combiner forms the following combined signals, $\tilde{x}_{1}$ and $\tilde{x}_{2}$ as

$$
\begin{aligned}
& \tilde{x}_{1}=h_{1}^{*} r_{1}+h_{2} r_{2}^{*} \\
& \tilde{x}_{2}=h_{2}^{*} r_{1}-h_{1} r_{2}^{*}
\end{aligned}
$$

Substituting for $h_{i}$ and $r_{i}$, the combined signals can be written as

$$
\tilde{x}_{1}=\left(\left|h_{1}\right|^{2}+\left|h_{2}\right|^{2}\right) x_{1}+h_{1}^{*} n_{1}+h_{2} n_{2}^{*}
$$




$$
\tilde{x}_{2}=\left(\left|h_{1}\right|^{2}+\left|h_{2}\right|^{2}\right) x_{2}-h_{1} n_{2}^{*}+h_{2}^{*} n_{1}
$$

In the absence of an error correcting code, these combined signals are sent to a maximum likelihood detector which for each estimated symbol $\tilde{x}_{1}$ chooses $x_{i}$ if and only if,

$$
d^{2}\left(\tilde{x}_{1}, x_{i}\right) \leq d^{2}\left(\tilde{x}_{1}, x_{k}\right)
$$

For $\mathrm{i} \neq \mathrm{k}$, where $d^{2}\left(\tilde{x}_{1}, x_{i}\right)$ is the Euclidean distance between the two symbols, assuming BPSK signals with an equal energy constellation.

\section{SYSTEM MODEL}

The system model of the Turbo Codes with Space-Time Transmit Diversity is shown in Figure 2. In this model, the sequence of information bits is encoded by the turbo encoder shown in Figure3. The turbo encoder is consists of two identical recursive systematic convolutional (RSC) component codes of rate $r$ which are separated by a pseudorandom interleaver. Thus, the same information sequence is encoded twice but in different orders. The choice of recursive systematic instead of feedforward convolutional codes is crucial for the performance of turbo codes. In general, the two component codes and their rates do not have to be the same. Without puncturing, this results in a rate $r=1 / 3$ turbo code. Note that this rate is achieved because each information bit is transmitted only once. The interleaver in Figure 3 decorrelates the inputs to the two decoders so that an iterative suboptimum decoding algorithm based on information exchange between the two component decoders can be applied. If the input sequences to the two component decoders are decorrelated there is a high probability that after the correction in one decoder the remaining errors should become correctable in the second decoder. The output sequence from the upper encoder, $\mathrm{C} 1$, and from the lower encoder, $\mathrm{C} 2$, are punctured and multiplexed to give the output of the encoder $\mathrm{C}$, the output of the encoder is modulated as shown in Figure 4. Prior to transmission, the modulated sequence $S$, is formatted by the space-time (ST) block encoder such that the transmitted sequences from the antennas are orthogonal to each other. As will be shown later, ST block codes in Figure4 can use two, three or four transmit antennas.

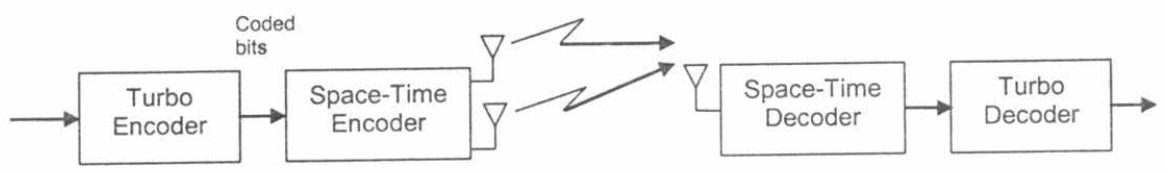

Fig. 2. Simplified system model of Turbo Codes with Space-Time Transmit Diversity. 


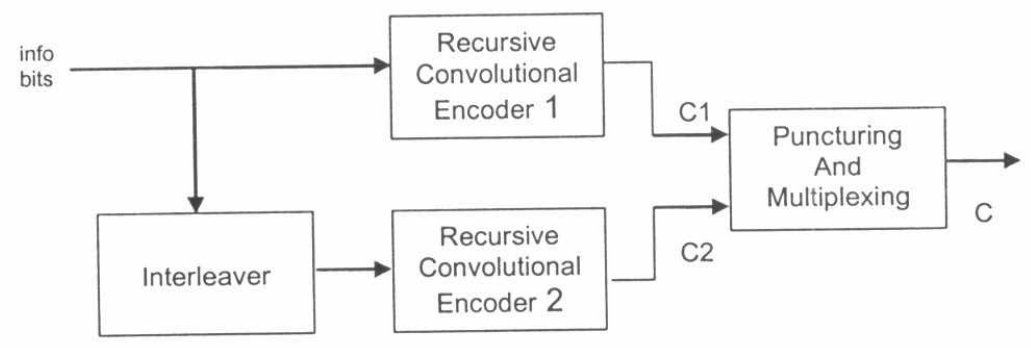

Fig. 3. Turbo Encoder.

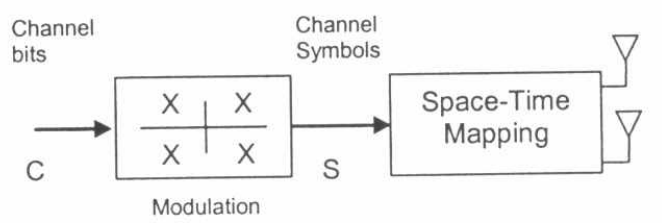

Fig. 4. Block diagram of a space-time block encoder.

\section{SIMULATION RESULTS}

This section contains the simulation results which illustrate the performance of binary turbo codes in combination with space time transmit diversity scheme. The performance is measured in terms of the bit error rate (BER) as a function of the bit energy to noise ratio Eb/NO. We scale down the transmit power from each antenna to maintain a unity total power With two identical component codes 4 -state $(1,5 / 7$ octal) and different puncturing patterns, the overall code is of a rate $(1 / 3,2 / 5,1 / 2,2 / 3)$. The $1^{\text {st }}$ Component code is terminated and the $2^{\text {nd }}$ is left open. A random interleaver with odd-even separation [13] is used. The output stream is BPSK modulated and sent through one, two, three, or four transmit antennas. A LOG-MAP decoder [12] with 8 iterations is employed at the receiver. A LOG-MAP decoder is similar to a maximum a-priori probability (MAP) decoder, but simpler since only addition and subtractions are performed [12].

The channel is subject to Rayleigh fading with fading coefficients being independent and identically distributed random variables (perfectly interleaved narrow-band or non-dispersive Rayleigh fading channel). It is assumed that the receiver had a perfect estimate of the channels' fading amplitudes. In all of the simulations, one receive antenna was used, and the total transmit power was constant and independent from the number of transmit antennas. The simulation program is written by MATLAB ${ }^{\text {TM }}$ version 6.5 . 


\subsection{Effect of the Number of Transmit Antennas}

Figure 5 shows the performance of the turbo code with one, two, three and four transmit antennas while keeping the total output power constant and doesn't depend on the number of the transmit antenna. The performance curves show that adding an extra transmit antenna increases diversity gain in the system which is shown in the different slopes of the curves. The higher the diversity gain the steeper the slope will be. Comparing with single transmit antenna, an improvement of $1.7 \mathrm{db}$ when using turbo codes with space time transmit diversity with no bandwidth expansion and only 2 antennas of half power, an improvement of $0.4 \mathrm{db}$ from 2 antennas to 3 antennas of $1 / 3$ power, and an improvement of $0.3 \mathrm{db}$ from 3 antennas to 4 antennas of $1 / 4$ power. So, there is a trade off between number of transmitting antennas and complexity.

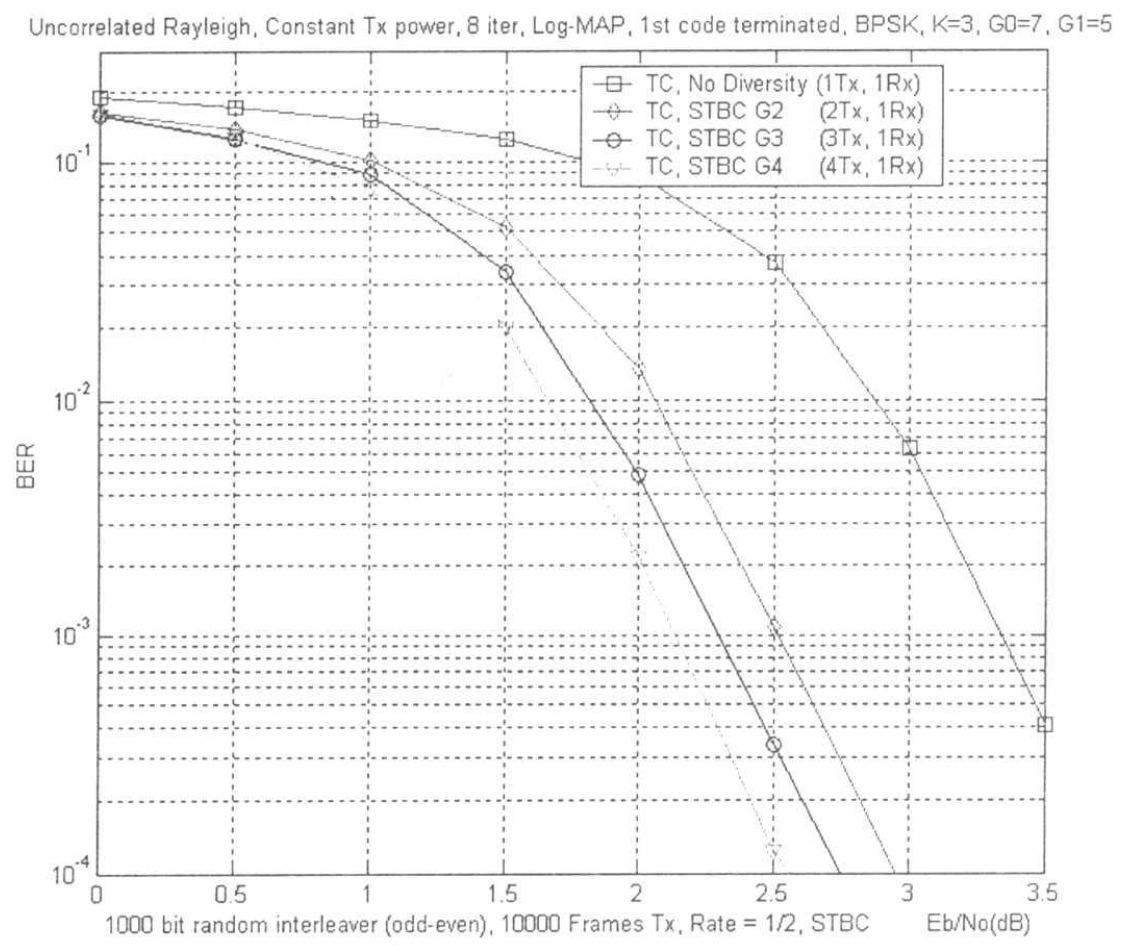

Fig. 5. Effect of the number of transmit antennas.

\subsection{Effect of Different Code Rates}

As described in section IV, in a turbo encoder two or more component encoders are used for generating parity information from an input data sequence. In this work we have used two RSC component encoders. Typically, in order to generate a half-rate code, half the parity bits from each component encoder are punctured. This was the arrangement used in their seminal paper by Berrou et al. on the concept of turbo codes [1]. However, it is of course possible to omit the puncturing and transmit all the 
parity information from both component encoders, which gives a one-third rate code. The performance of such a code, compared to the corresponding half-rate code, is shown in Figure 6. It can be seen that transmitting all the parity information gives a gain of about $0.9 \mathrm{~dB}$, in terms of Eb/NO, at a BER of $10^{-4}$.

Figure 6 shows the performance for higher code rates $2 / 5,1 / 2$, and $2 / 3$. From this figure, adding an extra parity bit enhancing the performance of the system. A similar gains are seen for turbo codes with different frame-lengths in the next section.

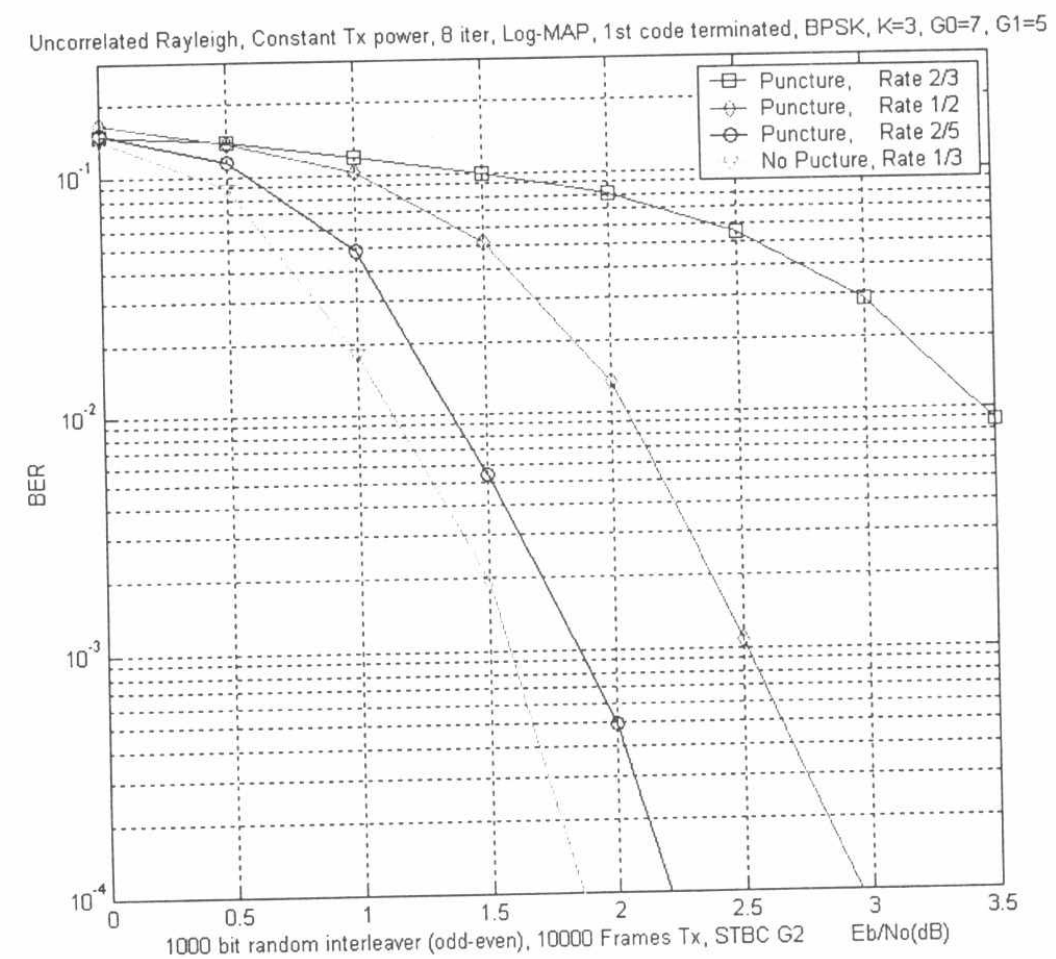

Fig. 6. Effect of different code rates.

\subsection{Effect of different Frame length}

Figure 7 shows a performance of turbo codes with 2 antennas and rate $1 / 3$ (no puncture). In the original paper on turbo coding by Berrou et al [1] and many of the subsequent papers, impressive results have been presented for coding with very large frame lengths. However, for many applications, for example in speech transmission systems, the large delays inherent in using high frame-lengths are unacceptable. Figure 7 shows how dramatically the performance of turbo codes depends on the frame-length $L$ used in the encoder. The 256 bit code would be suitable for use in a speech transmission system, while the 1024 bit code would be suitable for video transmission. The larger frame-length systems would be useful in data or non-real time transmission systems. It can be seen from Figure 7 that the 
performance of turbo codes is very impressive for systems with long frame lengths. However even for a short frame-length system, using 256 bits per frame, it can be seen that turbo codes give good results, and according to the application (voice, or data) we have different frame lengths (256, 512 or 1024 bits), Increasing the frame length from 256 to 512 and from 512 to 1024 brings about a $0.5 \mathrm{~dB}$ gain at a bit error rate $10^{-4}$, and the achievable gain is linearly proportional to the frame length.

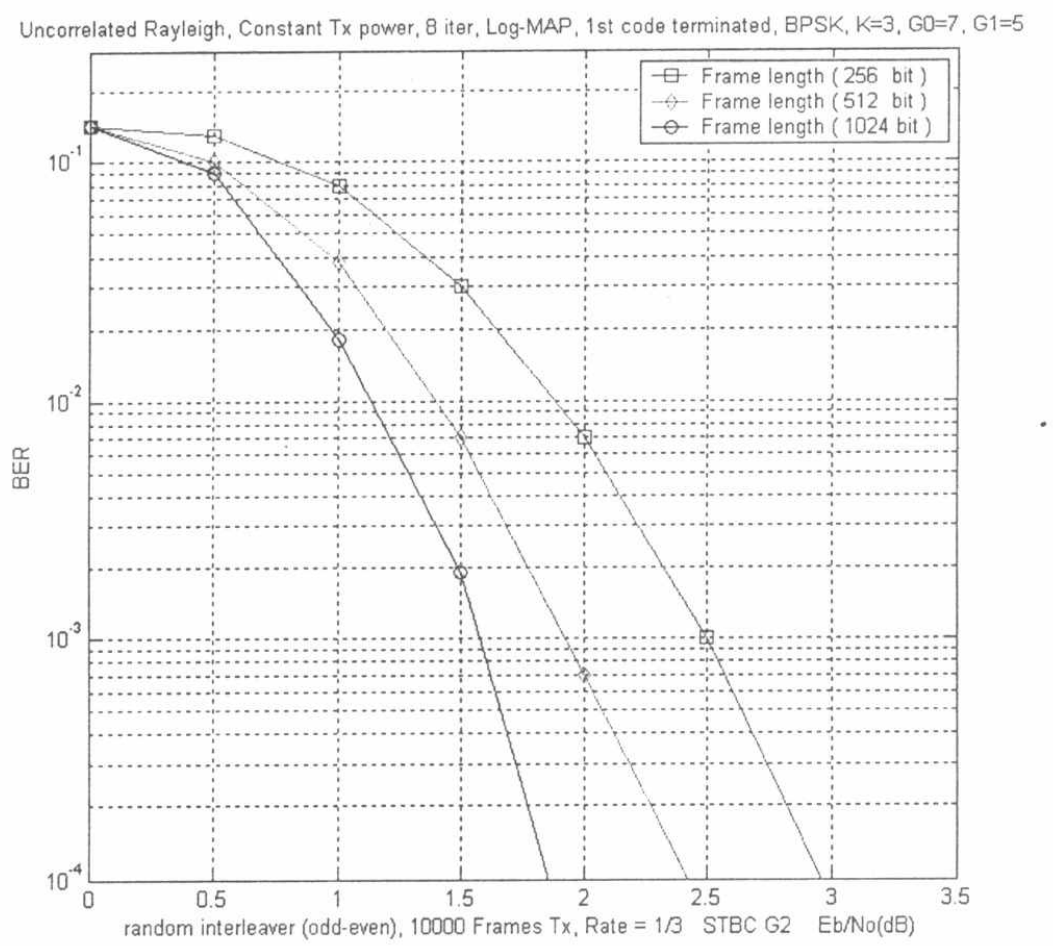

Fig. 7. Effect of different frame lengths.

\section{CONCLUSIONS}

In this paper, it is confirmed that transmit diversity techniques enhance the performance for binary turbo codes. Multiple transmit antennas satisfy a significant improvement in performance compared to the single antenna case. With real valued signals this improvement comes at no cost in terms of bandwidth expansion. . An improvement of $1.7 \mathrm{db}$ when using turbo codes with space time transmit diversity with no bandwidth expansion and only 2 antennas of half power was achieved. Performance at higher rates $2 / 5,1 / 2,2 / 3$ and different frame lengths were also investigated. 


\section{REFERENCES}

[1] C. Berrou, A. Glavieux, and P. Thitimajshima, "Near Shannon limit error-correcting coding and decoding: Turbo-codes," in Proceedings of ICC'93, pp. 1064-1070, 1993.

[2] 3rd Generation Partnership Project (3GPP), 3G TS 25.212, v3.5.0, Multiplexing and Channel Coding (FDD), Dec. 2000.

[3] E. K. Hall and S. G. Wilson, "Design and analysis of turbo codes on Rayleigh fading channels," IEEE J. Selected Areas Communications, vol. 16, pp. 160174, Feb. 1998.

[4] Jibing Wang, Marvin K. Simon "On the Performance of Space-Time Codes Over Spatially Correlated Rayleigh Fading Channels", IEEE Transactions on Communications, vol. 52, no. 6, June 2004.

[5] S. M. Alamouti, "A simple transmit diversity technique for wireless communications," IEEE J.Selected Areas in Communications, vol. 16, pp. 14511458, Oct. 1998.

[6] V. Tarokh, H. Jafarkhani, and A. Calderbank, "Space-time block codes from orthogonal designs", IEEE Trans. Inform. Theory, vol. 45, pp. 1456-1467, July 1999.

[7] B.Bjerke, Zoran Zvonar and John G. Proakis "Antenna Diversity Combining Schemes for WCDMA Systems in Fading Multipath Channels" , IEEE Transactions on Wireless Communications, vol. 3, no. 1, January 2004.

[8] S. L. Ariyavisitakul, "Turbo space-time processing to improve wireless channel capacity," IEEE Trans. on Communications, vol. 48, pp. 1347-1359, Aug. 2000.

[9] V. Tarokh, N. Seshadri, and A.R. Calderbank, "Space-time codes for high data rates wireless communications: Performance criterion and code construction," IEEE Trans. Inform. Theory, vol. 44, pp. 744- 765, 1998.

[10] G. Bauch, "Concatenation of space-time block codes and turbo-tcm," in Proc. IEEE Int. Conf. Communications, Vancouver, BC, Canada, June 1999, pp. 1202-1206.

[11] L. R. Bahl, J. Cocke, F. Jelinek, and J. Raviv, "Optimal decoding of linear codes for minimizing symbol error rate," IEEE Trans. on Information Theory, vol. IT-20, pp. 284-287, March 1974.

[12] P. Robertson, E. Villebrun, and P. Hoeher, "A comparison of optimal and suboptimal MAP decoding algorithms operating in the log domain," in Proceedings of ICC'95, pp. 1009-1013, 1995.

[13] A. Barbulescu and S. Pietrobon, "Interleaver design for turbo codes," IEE Electronics Letters, pp. 2107-2108,December 1994. 